\title{
Fabrication of Carbon Nanotube Tips for Scanning Tunneling Microscopy by Direct Growth Using the Microwave Plasma-Enhanced Chemical Vapor Deposition*
}

\author{
Kei Tanaka, ${ }^{\dagger}$ Masamichi Yoshimura, and Kazuyuki Ueda \\ Nano High-Tech Research Center, Toyota Technological Institute, \\ 2-12-1 Hisakata, Tempaku-ku, Nagoya 468-8511, Japan \\ (Received 11 November 2005; Accepted 4 March 2006; Published 18 March 2006)
}

\begin{abstract}
Carbon nanotube (CNT) tip for scanning tunneling microscopy (STM) is fabricated directly on a tungsten tip by microwave plasma-enhanced chemical vapor deposition (MPECVD). The reduction of the density of plasma ions directed to the apex of the tungsten tip suppresses the sputtering effect, and makes a successful fabrication of CNT tip. The length of grown CNTs can be well controlled below $1 \mu \mathrm{m}$ by growth time. The grown CNTs have bamboo-like structure with a catalyst attached on the top, as confirmed by transmission electron microscopy (TEM). Demonstrative observation of graphite surface on an atomistic scale is performed by the fabricated CNT tips. [DOI: 10.1380/ejssnt.2006.276]

Keywords: Scanning Tunneling Microscopy; Carbon Nanotube; Growth; Chemical Vapor Deposition; Plasma Processing
\end{abstract}

\section{INTRODUCTION}

One of promising applications of carbon nanotube (CNT) [1] is the use of nanoprobe in scanning probe microscopy (SPM), because it has small tip radius, high aspect ratio, mechanical robustness, chemical inertness and highly electrical conductivity. It has been reported that the CNT tips achieve higher lateral resolution than the conventional tips in tapping mode atomic force microscope (AFM) [2-4]. The mechanical behavior of CNTattached cantilever during the contact-mode AFM measurement has been investigated in detail $[5,6]$. The CNT has also been applied to scanning tunneling microscopy (STM) probes, where stable imaging of $\mathrm{Si}(111)[7], \mathrm{TaS}_{2}$ [2] and $\mathrm{Au}(111)$ [8] surface, comparable to the conventional metal probe, has been demonstrated at the atomic level. The conductive CNT probes would be useful in a multiprobe microscope [9]. Several fabrication methods of CNT tips have previously been demonstrated, for example, manual attachment of a CNT to the tip apex using an acrylic adhesive [2], electron beam induced deposition (EBID) of amorphous carbon under scanning electron microscope (SEM) [10], and direct growth by a chemical vapor deposition (CVD) technique [11-15].

The attachment of CNT using EBID of amorphous carbon under SEM is the most reliable method. As for CNT-STM tips, however, high contact resistance between the supporting tip and the attached CNT often becomes a problem. To overcome the problem, the metal coating method [16] and selective electron beam irradiation method [17] have been conducted. In contrast, the direct growth method using the CVD is likely to solve the problem, because the grown CNT is firmly in contact with a supporting tip. In addition, this direct growth method is quite suitable for the mass-scaled fabrication and automatically includes cleaning process of CNT itself.

In general, CVD is categorized by thermal and plasma process. By using plasma CVD for the fabrication of CNT

\footnotetext{
*This paper was presented at International Symposium on Surface Science and Nanotechnology (ISSS-4), Saitama, Japan, 14-17 November, 2005.

†Corresponding author: kei_tanaka@toyota-ti.ac.jp
}

tip, the CNT aligned parallel to the tip axis is possible, because the direction of incident ion of carbon species can be controlled by an applied electric field. However, the growth of CNT on the tip apex of small radius is extremely difficult due to the damage of plasma $[14,15]$. Yoshimura, et al. report successful fabrication of carbon nanostructures by reducing plasma damage [15]. However, the grown structure has a crystal habit and is unlikely to be a pure carbon nanotube. To the best of our knowledge, there are no reports that pure CNTs were fabricated on the STM tip by direct growth using plasma CVD.

In this paper, we report on the microwave plasmaenhanced CVD (MPECVD) growth of CNT on the STM tip apex. In order to suppress the sputtering of catalyst on the tip apex during the CVD process, the optimization of experimental condition of pressure is performed, and makes possible the growth of CNT on the tip apex. It was revealed from transmission electron microscopy (TEM) observation that the CNT show the bamboo-like structure with a catalyst existed on the top. Atomic resolution of graphite surface was obtained in STM images using the fabricated CNT tips.

\section{EXPERIMENTAL}

The growth of CNT on the tungsten tip was performed by using a commercially available MPECVD system (CVD-CN-100, Ulvac Japan). A tungsten tip was prepared by electrochemical etching in $\mathrm{KOH}$ solution. The iron thin film of $20 \mathrm{~nm}$, as the catalyst, was coated on the $\mathrm{W}$ tip by vacuum deposition. Prior to the Fe deposition, oxide layer covering the tip was removed by heating at $700^{\circ} \mathrm{C}$, which is a key process to assure the conductivity. It was then set into the small hole $(0.5 \mathrm{~mm} \phi)$ of the metal block in order to avoid the electric discharge from the plasma, and was placed on the electrode in the MPECVD system. The mixture of $\mathrm{CH}_{4}$ and $\mathrm{H}_{2}$ gases was used for the CVD growth. The flow ratio of $\mathrm{H}_{2}: \mathrm{CH}_{4}$ was kept constant at 3:2. Two kinds of total gas pressures were set at 226 and $67 \mathrm{~Pa}$. We used a microwave of $2.45 \mathrm{GHz}$ and $500 \mathrm{~W}$, and the growth times were 515 min. During the growth process, a voltage of $220 \mathrm{~V}$ was applied between the electrodes. Prior to the CNT 


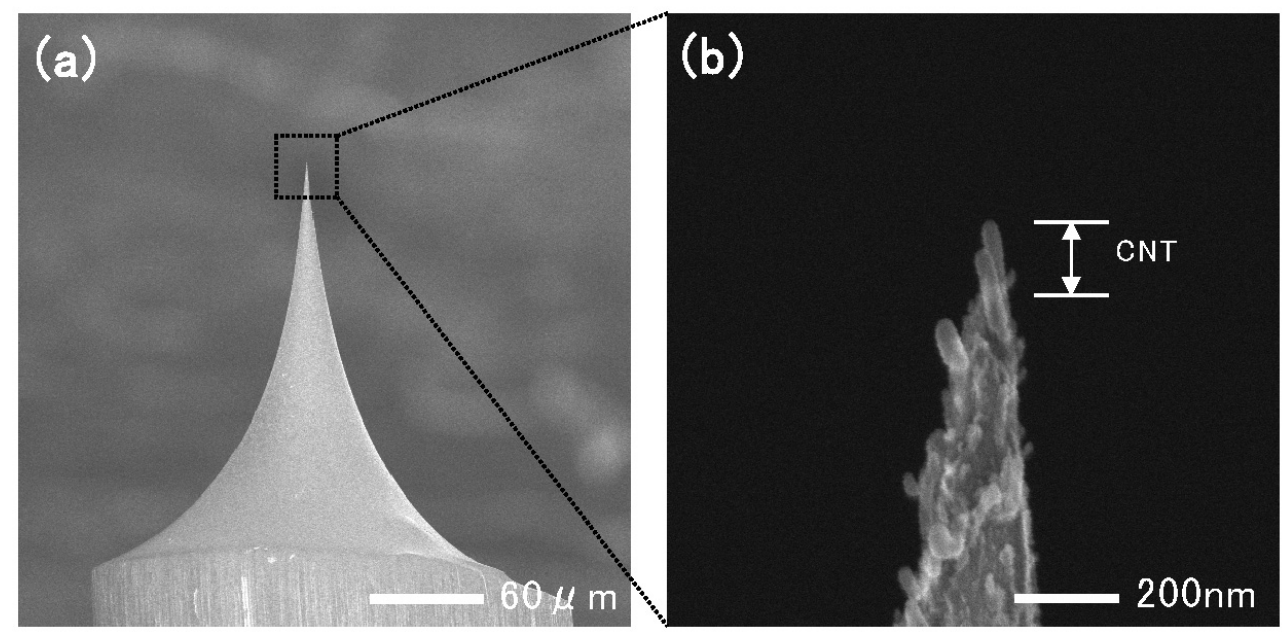

FIG. 1: (a) Low magnification SEM images of W Tip after CVD-growth. (b) High magnification SEM image of Tip apex. The pressure in the experimental conditions is $67 \mathrm{~Pa}$.

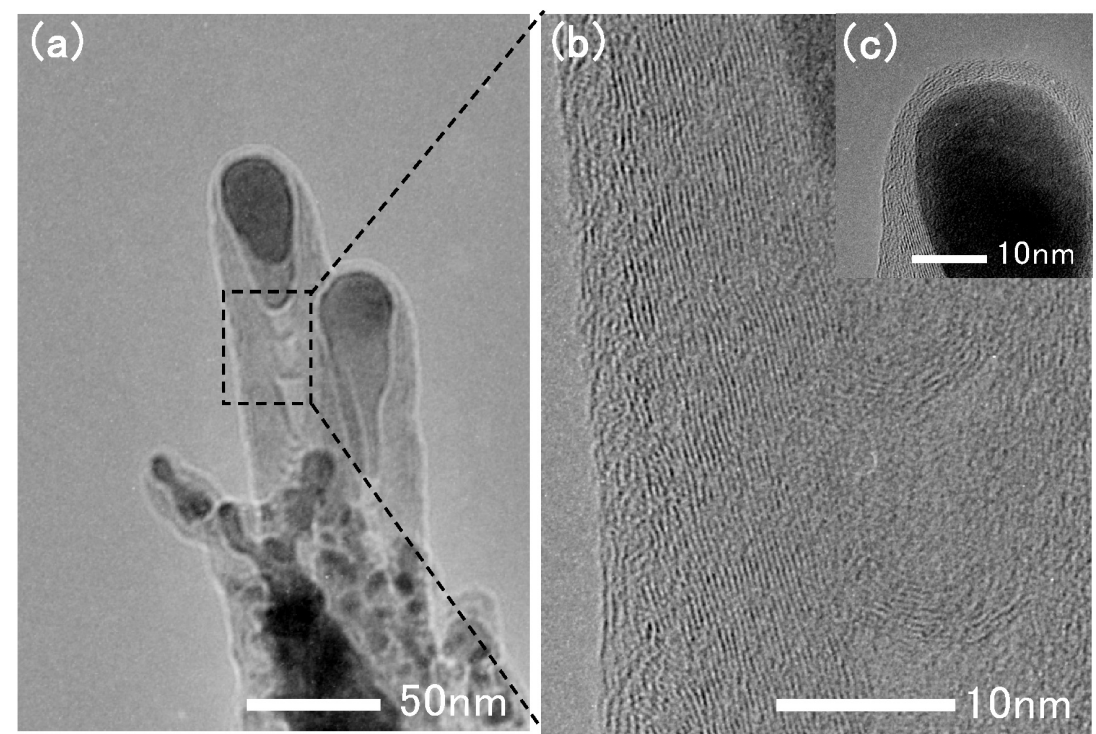

FIG. 2: (a) TEM image of CNTs on the tip apex. (b) High-resolution TEM images for the rectangular section in Fig. 2 (a). (c) Enlarged TEM image of catalyst particle.

growth, the tip was exposed to hydrogen plasma for the cleaning as well as activation of catalysts. During CVD process no heater was used, but the substrate temperature was raised to about $600^{\circ} \mathrm{C}$ as measured by thermocouple. The morphology of the tip after growth was observed by field emission scanning electron microscopy (FE-SEM, Hitachi, S4700). High-resolution transmission electron microscopy (TEM, JEOL, JEM2000EX) was carried out to determine the structure of the CNT. In order to examine the STM performance, a highly oriented pyrolytic graphite (HOPG) surface was observed in air.

\section{RESULTS AND DISCUSSION}

Figure 1(a) shows a SEM image of W tip after the CVD growth. The pressure was $67 \mathrm{~Pa}$. The CNTs of $\sim 40 \mathrm{~nm}$ in diameter and $\sim 140 \mathrm{~nm}$ in length have been grown on the tip apex as shown in Fig. 1(b). Figure 2 shows highresolution TEM images of the same tip of Fig. 1. The catalyst exists on the top of the CNT, and the structure of the CNT shows a so-called bamboo-like. The sidewall of CNT consists of graphen sheets of about 30 layers (Fig. $2(\mathrm{~b}))$. The top of the catalyst is coated with amorphous carbon of about $3 \mathrm{~nm}$ in thickness (Fig. 2(c)). It was reported that the amorphous carbon tends to form at lower growth temperatures [18]. It is noted here that pure CNTs can be fabricated onto the tip apex by plasma CVD for the first time.

In contrast, no CNTs growth on the tip apex was observed at a higher pressure of $226 \mathrm{~Pa}$, which is standard condition for growth of CNTs using this MPECVD system. In place, growth of CNT was observed only at the root of metal tip. This tendency is basically similar to that reported by Pan, et al. [14] and Yoshimura, et al. [15]. Since the ion flux at the tip apex is higher than that 

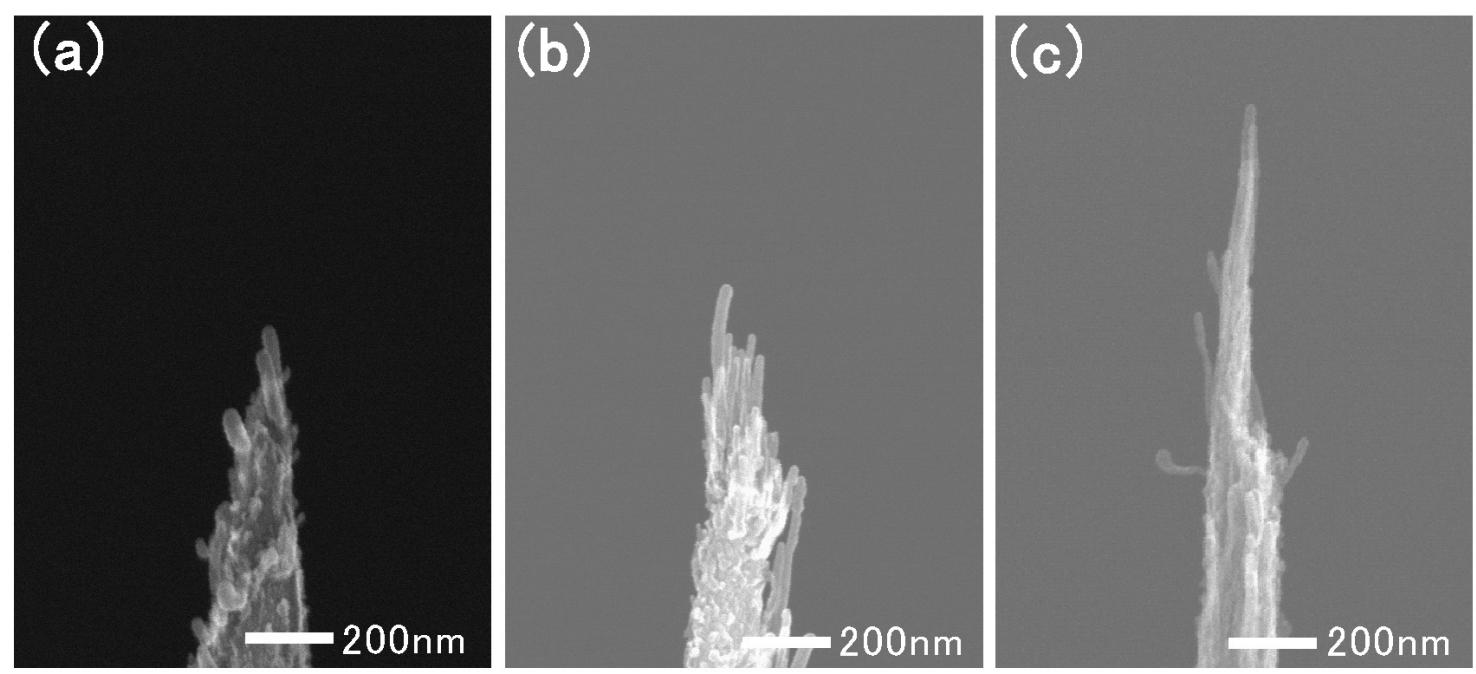

FIG. 3: SEM images of CNTs grown at three different growth time, (a) 5 min, (b) 7 min, (c) 10 min.

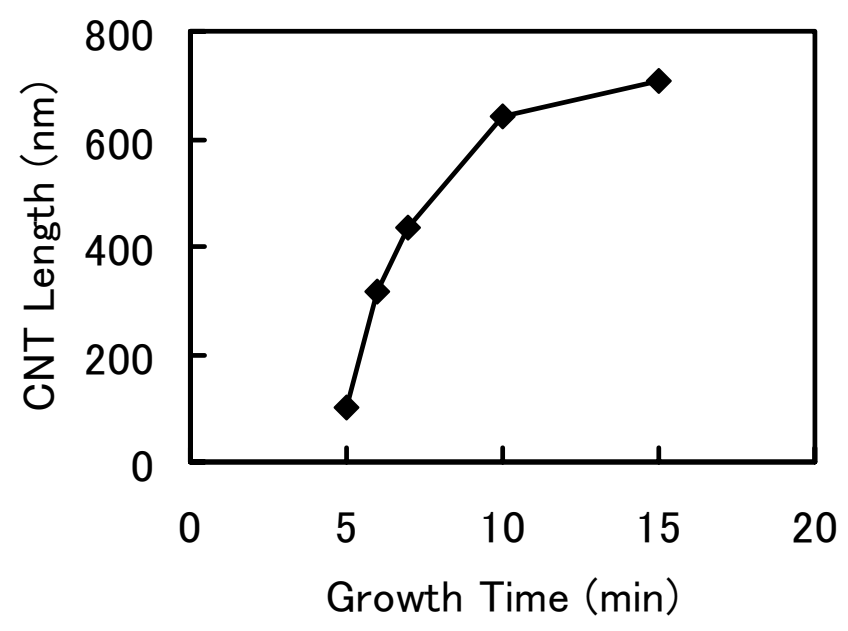

FIG. 4: Growth time dependence of CNTs length.

at the root due to different electric field concentration, Fe catalyst would be sputtered away preferentially from the tip apex by the ion bombardment. Thus low-density plasma due to the pressure reduction suppresses the sputtering, and makes successful fabrication at the apex, as shown in Fig. 1.

Figure 3 shows CNTs grown at three different growth time. The grown CNTs are well oriented along the axis of the tungsten wire. This is one of merits in the use of plasma-enhanced CVD where bias voltage plays a role to attract ion species. The length and the number of CNTs increase with growth time. The bundle formation of CNTs occurs during growth as shown in Fig. 3(c), which is consistent with previous report [19]. Figure 4 shows the length of CNTs as a function of growth time. The growth rate gradually decreases with growth time, and then the length is saturated with $\sim 800 \mathrm{~nm}$. The decrease in growth rate is attributed to the fact that the thickness of carbon film on the top of catalyst particle increases with growth time. Consequently, it became more difficult for the reactive carbon species to reach the cat-

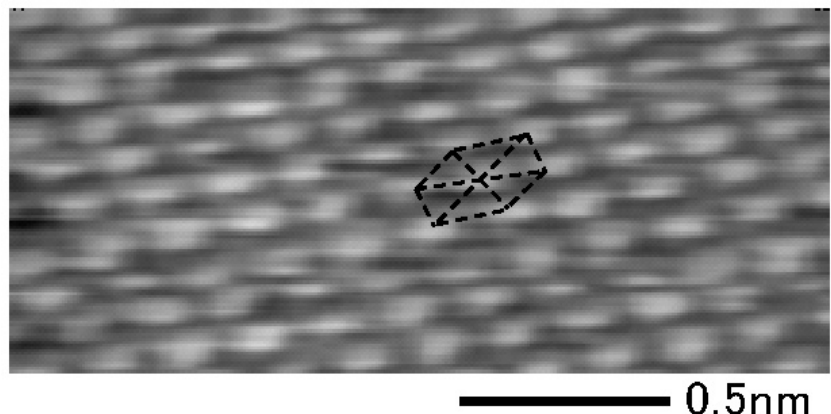

FIG. 5: STM image of a HOPG surface obtained using the fabricated CNT Tip.

alyst particle. The aspect ratios of the fabricated probes here are estimated to range from 1:3 to 1:30 depending on the growth time. These values are almost the same as reported ones $[7,10,16]$. The tip of high aspect ratio, as shown in Fig. 3(c), can be used for high roughness sample as well as for multiprobe use. The tip with lower aspect ratio, as shown in Fig. 3(a), can be used for electron beam source or atom manipulation.

Lastly we demonstrate the performance of the CNT tip fabricated in the present study. Figure 5 shows a STM image of HOPG surface using the fabricated CNT tip, at a bias voltage of $0.08 \mathrm{~V}$ and a tunneling current of $0.7 \mathrm{nA}$. A periodic structure is observed. Thus it is confirmed that the fabricated CNT tip achieves atomic resolution. Before use for STM, however, the electronic structure of the tip should be clarified because the STM image results from the convolution of electronic structures of tip and sample surface. Scanning tunneling spectroscopy measurement would be necessary to clear this point in the future.

\section{CONCLUSIONS}

In conclusion, we have fabricated CNT tips for STM by direct growth using MPECVD. Low ion density in plasma 
was essential factor for the growth of CNTs, and sputtering of catalysts on the tip apex should be suppressed. The CNTs length can be controlled by growth time. TEM images revealed a bamboo-like structure with a catalyst attached on the top of CNTs, which would be expected as novel magnetic force microscopy (MFM) tips. The atomic images of HOPG are obtained in the STM observation using the fabricated CNT tip. This fabrication method is expected as mass-scaled fabrication of CNT tip.

\section{Acknowledgments}

The authors thank to Dr. A. Okamoto (Toyota Central R \& D Lab., Inc.) for his help to TEM observation. This work is supported by the High-Tech Research Center project for Private Universities: matching fund subsidy from the Ministry of Education, Culture, Sports, Science and Technology (MEXT), 2001-2005.
[1] S. Iijima, Nature 354, 56 (1991).

[2] H. Dai, J. H. Hafner, A. G. Rinzler, D. T. Colbert and R. E. Smally, Nautre 384, 147 (1996).

[3] S. S. Wong, A. T. Wooley, T. W. Odom, J. H. Huang, P. Kim, D. V. Vezenov and C. M. Lieber, Appl. Phys. Lett. 73, 3465 (1998).

[4] J. H. Hafner, C. L. Cheung, T. H. Oosterkamp and C. M. Lieber, J. Phys. Chem. B 105, 743 (2001).

[5] M. Ishikawa, M. Yoshimura and K. Ueda, Appl. Surf. Sci. 188, 456 (2001).

[6] M. Ishikawa, M. Yoshimura and K. Ueda, Physica B 323, 184 (2002).

[7] T. Shimizu, H. Tokumoto, S. Akita and Y. Nakayama, Surf. Sci. 486, L455 (2001).

[8] W. Mizutani, N. Choi, T. Uchihashi and H. Tokumoto, Jpn. J. Phys. 40, 4328 (2001).

[9] M. Ishikawa, M. Yoshimura and K. Ueda, Jpn. J. Appl. Phys. 44, 1502 (2005).

[10] H. Nishijima, S. Kamo, S. Akita, Y. Nakayama, K. I. Hohmura, S. H. Yoshimura and K. Takeyasu, Appl. Phys. Lett. 74, 4061 (1999).

[11] C. L. Cheung, J. H. Hafner, T. W. Odom, K. Kim and C.
M. lieber, Appl. Phys. Lett. 76, 3136 (2000).

[12] E. Yenilmez, Qian Wang, R. J. Chen, D. Wang and H. Dai, Appl. Phys. Lett. 80, 2225 (2002).

[13] Y. Shingaya, T. Nakayama and M. Aono, Physica B 323, $153(2002)$

[14] F. M. Pan, Y. B. Liu, Y. Chang, C. Y. Chen, T. G. Tsai, M. N. Chang and J. T. Sheu, J. Vac. Sci. Technol. B 22, 90 (2004).

[15] M. Yoshimua, S. Jo and K. Ueda, Jpn. J. Appl. Phys. 42, 4841 (2003).

[16] T. Ikuno, M. Katayama, M. Kishida, K. Kamada, Y. Murata, T. Yasuda, S. Honda, J. Lee, H. Mori and K. Oua, Jpn. J. Appl. Phys. 43, L644 (2004).

[17] A. Ando, T. Shimazu, H. Abe, Y. Nakayama and H. Tokumoto, Physica E 24, 6 (2004).

[18] M. Chhowalla, K. B. K. Teo, C. Ducati, N. L. Rupesinghe, G. A. J. Amaratunga, A. C. Ferrari, D. Roy, J. Robertson and W. I. Milne, J. Appl. Phys. 90, 5308 (2001).

[19] H. Sato, H. Takegawa and Y. Saito, J. Vac. Sci. Technol. B 21, 2564 (2003). 